\title{
Multiaxial yield behaviour of polypropylene
}

\author{
M. Jerabek ${ }^{1, a}$, D. Tscharnuter ${ }^{2}$, Z. Major ${ }^{3}$, K. Ravi-Chandar ${ }^{4}$, R. W. Lang ${ }^{5}$ \\ ${ }^{1}$ Borealis Polyolefine GmbH, St. Peter Str. 25, 4021 Linz, Austria \\ ${ }^{2}$ Polymer Competence Center Leoben GmbH, Roseggerstr. 12, 8700 Leoben, Austria \\ ${ }^{3}$ Institute of Polymer Materials, Altenberger Str 69, Johannes Kepler University Linz, Austria \\ ${ }^{4}$ Center for Mechanics of Materials, Solids and Structures, The University of Texas at Austin, 1 \\ University Station, TX 78712, USA \\ ${ }^{5}$ Institute of Polymer Product Engineering, Altenberger Str 69, Johannes Kepler University Linz, \\ Austria
}

\begin{abstract}
In order to characterize the yield behavior of polypropylene as a function of pressure and to verify the applicability of the Drucker-Prager yield function, various tests were conducted to cover a wide range of stress states from uniaxial tension and compression to multiaxial tension and confined compression. Tests were performed below and above the glass transition temperature, to study the combined effect of pressure and temperature. The pressure sensitivity coefficient as an intrinsic material parameter was determined as a function of temperature. Increasing pressure sensitivity values were found with increasing temperature, which can be related to the change in the free volume and thus, to the enhanced molecular mobility. A best-fit Drucker-Prager yield function was applied to the experimental yield stresses and an average error between the predictions and the measurements of $7 \%$ was obtained.
\end{abstract}

\section{Introduction}

In many engineering applications polymeric materials are exposed to complex multiaxial loading situations where in addition to tensile loads, compressive stresses may be dominant. Typical examples of multiaxial compressive loadings in practical applications of polymers include structural components exposed to direct compressive or bending loads like rollers, gears, bearings, damping materials and other similar applications. On a local scale, complex compressive stresses also play a major role in joint technologies (e.g., screw joints, clamping, fastening), in cutting processes and in tribological applications (i.e., friction, wear and scratch processes) also including fretting fatigue loads [1-4]. Another example for the development of multiaxial local stresses is in the area of polymer matrix composites, in which a multiaxial stress field develops on a local scale around the reinforcing phase, even though the external load applied to the material may be a uniaxial tensile load [5]

Considering that the behavior of polymeric materials under tension and compression may differ particularly in the yield and post-yield regime [6-8], the need to characterize the whole range of stress states of plastics becomes evident. Moreover, the above examples indicate the importance of

a e-mail : michael.jerabek@borealisgroup.com

This is an Open Access article distributed under the terms of the Creative Commons Attribution-Noncommercial License 3.0, which permits unrestricted use, distribution, and reproduction in any noncommercial medium, provided the original work is properly cited. 
adequate test set-ups in a comprehensive manner and underline the need for appropriate test methodologies. Last but not least, adequate material data and material laws for multiaxial loadings are especially needed in advanced component design procedures and for structural reliability assessments based on modern simulation tools.

For the description of the 3D yielding behavior of polymeric materials, the whole range of stress loadings must be applied to pressure dependent materials, in which the yield surface is shifted to larger values in the compression regime than those in the tensile domain $[9,10]$. In recent studies the Drucker-Prager yield criterion [11] was successfully applied to polymeric materials $[12,13]$ and thus also chosen in this investigation. In order to acquire sufficient experimental data for the evaluation of the yield surface, various test methods covering the whole stress range from biaxial and uniaxial tensile tests in the low pressure regime to fully confined compression tests in the high pressure regime were conducted.

\section{Materials and Experimental}

The material investigated in this study was a development grade polypropylene (PP) homopolymer, manufactured and delivered by Borealis Polyolefine GmbH (Linz, A) either as injection molded plates, out of which all specimens were machined for the torsion test, biaxial tensile test and the various compression test set-ups, or as injection molded tensile specimens according to ISO 3167 , type B. All tests were carried out at a strain rate of $8.7 \times 10^{-4} \mathrm{~s}^{-1}$ and at ambient temperature. Tests at $80{ }^{\circ} \mathrm{C}$ and $-30{ }^{\circ} \mathrm{C}$ were performed for the various compression test set-ups and for the uniaxial tensile test. For all test set-ups only a brief introduction is given, as the reader is referred to the literature cited for an in-depth explanation.

The biaxial bulge test was carried out, to investigate the behavior of the material in biaxial tension [14]. A round thin sheet of the material with a diameter of $140 \mathrm{~mm}$ and a thickness of $1 \mathrm{~mm}$ is clamped between two fixing grips and pressurized on one side by air, measuring simultaneously the exact pressure and the effective strain in the middle of the specimen system utilizing a digital image correlation (DIC) system. In the center zone of the specimen a true biaxial stress state is guaranteed and was checked at every measurement by the optical strain measurement device. The uniaxial tensile tests were performed on an electro-mechanical universal testing machine of the type Instron 5500 (Instron LTD; High Wycombe, UK). The initial specimen length between the wedge grips was $115 \mathrm{~mm}$. The axial strain was measured by a mechanical clip-on extensometer of the type Instron 2630-112 with an initial gage length of $50 \mathrm{~mm}$. A temperature chamber was mounted on the frame of the testing machine to carry out tests at $-30{ }^{\circ} \mathrm{C}$ and at $80^{\circ} \mathrm{C}$.

In order to characterize the torsion behavior of $\mathrm{PP}$, an axial/torsional servo-hydraulic test system of the type MTS 359 (MTS Systems GmbH; Berlin, D) was utilized for these experiments, using the unnotched round bar specimen. The angle of twist, which is proportional to the shear strain, and the torque were measured to determine the shear strain vs. shear stress. In order to characterize the compression behavior of polymeric materials, uniaxial and confined compression experiments were carried out using a recently developed compression device with aligning bars at each corner [15]. Due to the four ball linings and highly accurate aligning bars, a precise and reproducible movement of the upper and lower compression plate is guaranteed and any transverse forces and moments are avoided. As to the accurate determination of axial strain, an LVDT mounted between the two compression plates was used. Again, all tests were carried out on the electro-mechanical driven universal testing machine of the type Instron 5500 (Instron LTD; High Wycombe, UK) also using the temperature chamber for non-ambient test temperatures.

As described in detail in [15], highly polished plates and a PTFE lubricant were used to minimize the effect of friction between the specimen surface and the compression plates. A uniform specimen deformation in the pre-yield regime up to the yield point was obtained, which was of prime interest for this investigation. For the determination of the true stress-true strain curve, a constant volume during deformation was assumed $(v=0.5)$, as significant differences to the true stress values 
calculated taking the experimentally measured Poisson's ratio are only noticeable in the post-yield but not in the pre-yield regime.

The multiaxial confined compression test method used for this study corresponds to a method described in detail by $\mathrm{Ma}$ and Ravi-Chandar [16] and Qvale and Ravi-Chandar [17]. In this configuration the specimen is surrounded by a confining cylinder, which restrains the free lateral expansion during axial loading without prohibiting it totally. Assuming a homogeneous deformation of the cylindrical specimen and a perfect fit between the specimen and the cylinder, the stress and strain field within the specimen and cylinder can be calculated via the Lamé solution. The measured values include the longitudinal force, local displacement provided by an LVDT, and the hoop strain of the confining cylinder determined via strain gauges mounted on the outer cylinder surface. By varying the thickness of the cylinder or by using a cylinder material of a different modulus, the stress state applied on the specimen can be changed and accordingly adapted. Of special importance, this configuration allows for an inelastic as well as a viscoelastic characterization of polymers without a priori knowing the constitutive equation $[17,18]$. Furthermore, while in uniaxial compression testing an inhomogeneous specimen deformation may occur at high strains, in confined compression the strain localization is significantly reduced or yet eliminated.

In uniaxial and confined compression test set-ups, cylindrical specimens of $20 \mathrm{~mm}$ in length, $l$, and of $10 \mathrm{~mm}$ in diameter, $2 a$, were used. It should be pointed out that specimens used for torsion, uniaxial and confined compression tests were machined from the $13 \mathrm{~mm}$ thick injection molded plates along the flow direction to avoid effects of different molecular orientations in the specimens. Confining cylinders of various diameters were produced out of rods made of a commercial polypropylene with outer diameters of $12 \mathrm{~mm}, 15 \mathrm{~mm}, 20 \mathrm{~mm}, 30 \mathrm{~mm}$ and $42 \mathrm{~mm}$. For the analysis of the stress and strain field in the specimen, the mechanical behavior of the confining cylinder was determined via uniaxial tensile tests. The specimens were machined from the rods and tests were performed at the three different temperatures $\left(23{ }^{\circ} \mathrm{C}, 80^{\circ} \mathrm{C},-30{ }^{\circ} \mathrm{C}\right)$.

\section{Yield Point and Effective Stress Definition}

Different approaches are described in the literature [19-22] for the identification of the yield point in a monotonically increasing stress-strain curve. While several methods have no physical background (e.g., offset method, bilinear fit of the stress-strain curve), strain recovery experiments may predict the onset of yielding accurately based on irreversible deformation of the specimen [23]. As the number of test specimens increases significantly for the latter approach, a simple method was applied in this study. In uniaxial tension test, the first peak value in the stress-strain relationship was taken as the nominal yield point. In uniaxial compression the true stress-true strain curve was utilized to identify the strain at yield. Only slightly larger yield (true) strain values were identified in compression than in tension tests. Due to the limited dependence of the yield strain on pressure, the yield strain values obtained in uniaxial compression were assumed as the yield strain also in the confined compression experiments.

To allow a comparison of yield stresses obtained under different loading conditions, the stress state is basically divided into a hydrostatic and deviatoric (equivalent) part, where the latter is actually responsible for the plastic deformation of the material. In this work the equivalent stress and equivalent strain definitions of Kachanov [24] were applied which are given by

$$
\begin{gathered}
\tau_{e}=\frac{1}{\sqrt{6}} \sqrt{\left(\sigma_{z z}-\sigma_{r r}\right)^{2}+\left(\sigma_{r r}-\sigma_{\theta \theta}\right)^{2}+\left(\sigma_{\theta \theta}-\sigma_{z z}\right)^{2}+6\left(\tau_{z r}^{2}+\tau_{r \theta}^{2}+\tau_{\theta z}^{2}\right)} \\
\gamma_{e}=\sqrt{\frac{2}{3}\left[\left(\varepsilon_{z z}-\varepsilon_{r r}\right)^{2}+\left(\varepsilon_{r r}-\varepsilon_{\theta \theta}\right)^{2}+\left(\varepsilon_{\theta \theta}-\varepsilon_{z z}\right)^{2}+\frac{3}{2}\left(\gamma_{z r}^{2}+\gamma_{r \theta}^{2}+\gamma_{\theta z}^{2}\right)\right]}
\end{gathered}
$$


where $\tau_{e}$ and $\gamma_{e}$ are the equivalent stress and strain, respectively. Relating the effective stress to the mean pressure acting on the specimen at the yield point, may in most cases provide a linear correlation with the slope being the pressure sensitivity index $\alpha$ according to

$$
\tau_{e}=\tau_{0}+\alpha \sigma_{m}
$$

where $\tau_{0}$ is the effective stress at zero pressure (torsion test).

\section{Results and Discussion}

True stress - true strain curves of uniaxial tensile, uniaxial compressive and multiaxial compressive tests conducted at the three investigated temperatures are depicted in Fig. 1. The tensile test is plotted up to the nominal yield point, thus the end of the curve does not necessarily represent ultimate failure of the specimen. Confined compression experiments were conducted to a value of longitudinal strain of $15 \%$, as up to this level the material does not get squeezed out between the loading pin and the confining cylinder. Beyond the initial seating effects seen in the two compression set-ups, the stress-strain curves surpass that of tension and considerably larger stress levels were observed depending on the level of confinement. This emphasizes the importance of characterizing polymeric materials under adequate testing conditions by applying proper constraint conditions and illustrates the wide range of mechanical behavior of PP. As to the determination of the yield stress, the simple approach described above was applied. In the post-yield regime, strain softening was observed in uniaxial compression, while no strain softening was determined for any of the $b / a$ ratios in the confined compression set-up, yet the tendency for strain hardening increases with higher constraint level.

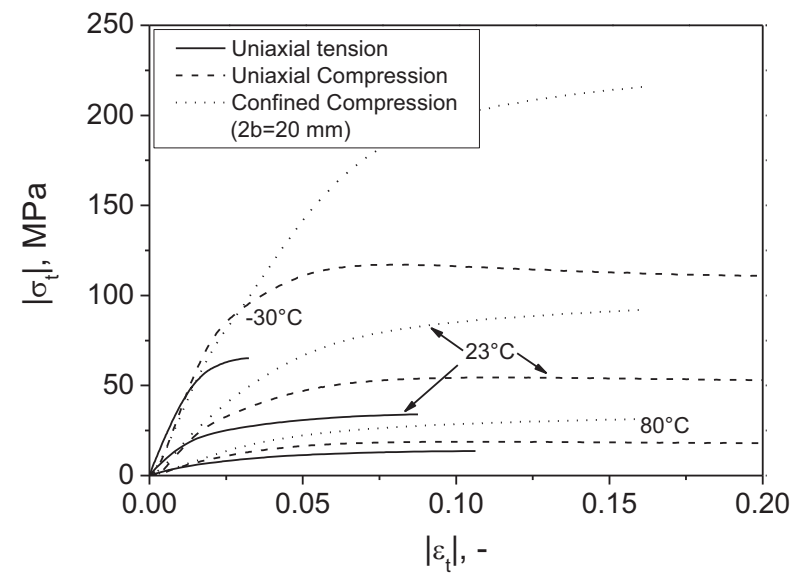

Fig. 1. Comparison of true stress-true strain curves of uniaxial tension, uniaxial compression and confined compression for all temperatures studied. 
In principle, not only the yield stress but all mechanical properties in general of polymeric materials i.e., modulus, Poisson's ratio, strain at break, etc. depend on hydrostatic pressure [25]. The equivalent stress calculated at the respective yield point is depicted in Fig. 2 as a function of mean stress. The pressure range covers a wide set of experiments from biaxial tension to fully confined compression. A linear relation between the equivalent stress and pressure was obtained for PP. This finding is in agreement to what is assumed in many publications to be the intrinsic material behavior $[26,27]$, but it is worth noting that bilinear and even nonlinear correlations have also been reported, depending on the stiffness, the molecular weight, and the crystallinity of the polymer [6, 7]. Moreover, the linear fit to the experimental data also shown in Fig. 2 provides the pressure sensitivity index, an intrinsic material parameter used as input parameter for pressure dependent yield criteria [12].

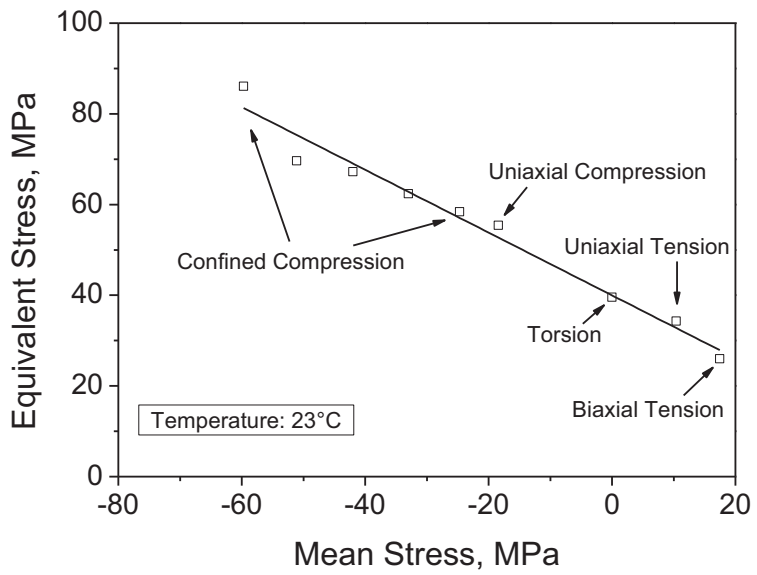

Fig. 2. Equivalent stress as a function a function of mean stress for $23{ }^{\circ} \mathrm{C}$.

The significant effect of pressure is related to the decrease of free volume and thus, to the reduced molecular mobility. As the free volume is frozen below $\mathrm{T}_{\mathrm{g}}$ and increases linearly with temperature above $\mathrm{T}_{\mathrm{g}}[28]$, a significantly enhanced pressure dependence may be expected at $80{ }^{\circ} \mathrm{C}$. On the other hand, the pressure sensitivity should decrease for test temperatures below $\mathrm{T}_{\mathrm{g}}$, which is the case for a test temperature of $-30{ }^{\circ} \mathrm{C}$. For each of the two non-ambient test temperatures an excellent linear correlation was obtained, which indicates that in the investigated pressure range only one molecular relaxation process is dominant.

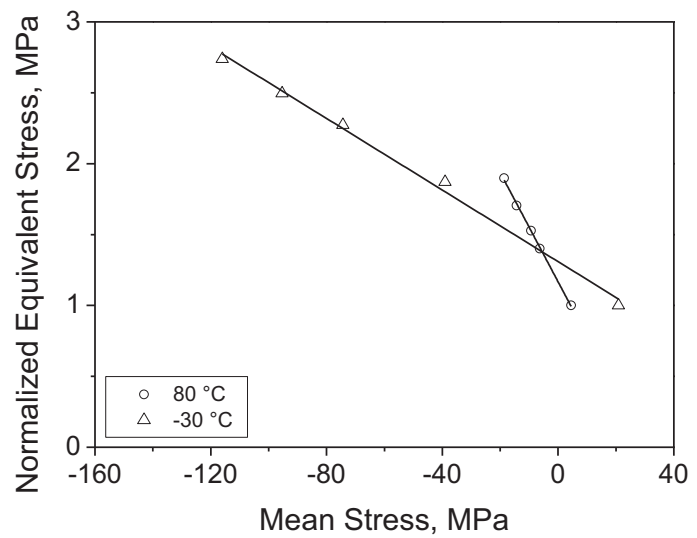

Fig. 3. Normalized equivalent stress plotted vs. mean stress for non-ambient temperatures.

In order to determine the effect of pressure as a function of temperature, normalized equivalent stresses were calculated based on the tensile equivalent stress. This procedure enables to calculate 
the pressure sensitivity coefficient free of absolute values and allows for a determination of the intrinsic material behavior. Moreover, it facilitates a "true" comparison of different temperatures. In Fig. 3 the normalized equivalent stress is plotted against the mean stress for the two non-ambient test temperatures. In accordance with the explanation above, while a significantly steeper slope of the linear fit was obtained for the elevated test temperature, reduced pressure dependence was noticed for $-30{ }^{\circ} \mathrm{C}$. This "normalized" pressure sensitivity coefficient is plotted in Fig. 4 as a function of temperature. An overall increase of the pressure dependence by a factor of 4 was determined in the investigated temperature range. An appropriate function may be used to describe the pressure dependence on temperature, but for an unambiguous correlation obviously more test temperatures are necessary.

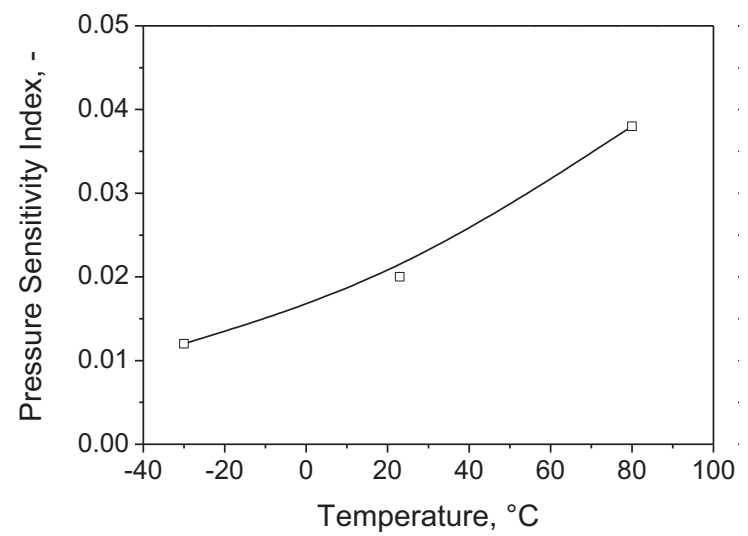

Fig. 4. Pressure sensitivity index as a function of temperature.

As to the prediction of the yield behavior of polymeric materials for arbitrary stress states, the pressure dependent 3D yield criterion of Drucker - Prager was utilized. This criterion can be given in the form

$$
\sigma_{e}+\sigma_{m} \tan \alpha-\left(1+\frac{\tan \alpha}{3}\right) \sigma_{c}=0
$$

where $\sigma_{e}=\sqrt{3} \tau_{e}$ is the von Mises effective stress, and $\sigma_{c}$ is the yield stress under uniaxial compression. If the pressure sensitivity index is zero, we recover the von Mises yield condition. The Drucker Prager yield function was fitted to the experimental test results and a best fit value of the pressure sensitivity index of about $31.5^{\circ}$ was obtained, which is remarkably higher than those obtained for amorphous materials and for PP described in the literature $[12,26]$. Note that the pressure sensitivity indexes determined via Eq. 3 and 4 are two independent values and cannot be compared to each other.

The resulting yield surface and the experimentally measured yield stresses in the various test setups are depicted in Fig. 5. The yield surface described by the Drucker-Prager criterion has a conical shape in the direction of but not axially symmetric to the hydrostatic axis. The criterion provides significantly lager yield stresses in the compressive than in the tensile regime. In fact, plastic deformation of the material is predicted under triaxial tensile loading conditions at a stress value of $72 \mathrm{MPa}$, while yielding under fully multiaxial compressive loading is prohibited. Intersection of the yield criterion with the plane-stress plane is also plotted, indicating the excellent agreement with the measured values. As the Drucker-Prager yield criterion has a conical shape, intersection with the plane-stress plane provides an ellipsoidal 2D yield surface, which is comparable to the modified von Mises yield criterion. The multiaxial confined compression test results are also shown in the 3D stress space along with the best-fit lines from the conical yield surface through the obtained multiaxial test results. As to the accuracy of the prediction capabilities of the yield criterion, an average error between the experimental yield stresses and the calculated values of about $8 \%$ was 
found. To provide a complete description of the yield surface, suitable fitting functions may be applied to the pressure sensitivity coefficient as a function of temperature (see Fig.4) and strain rate to describe their behavior. In general, all parameters affecting the mechanical behavior of polymeric materials can be taken into consideration, like physical aging. The effective parameters (loading conditions) do not simply shift the yield surface to larger or smaller values, respectively, but may also change the slope of the yield surface remarkably. Including additional parameters into the yield criterion, may allow for a complete description of the multiaxial yielding behavior of a viscoelastic material for arbitrary loading history and material state.

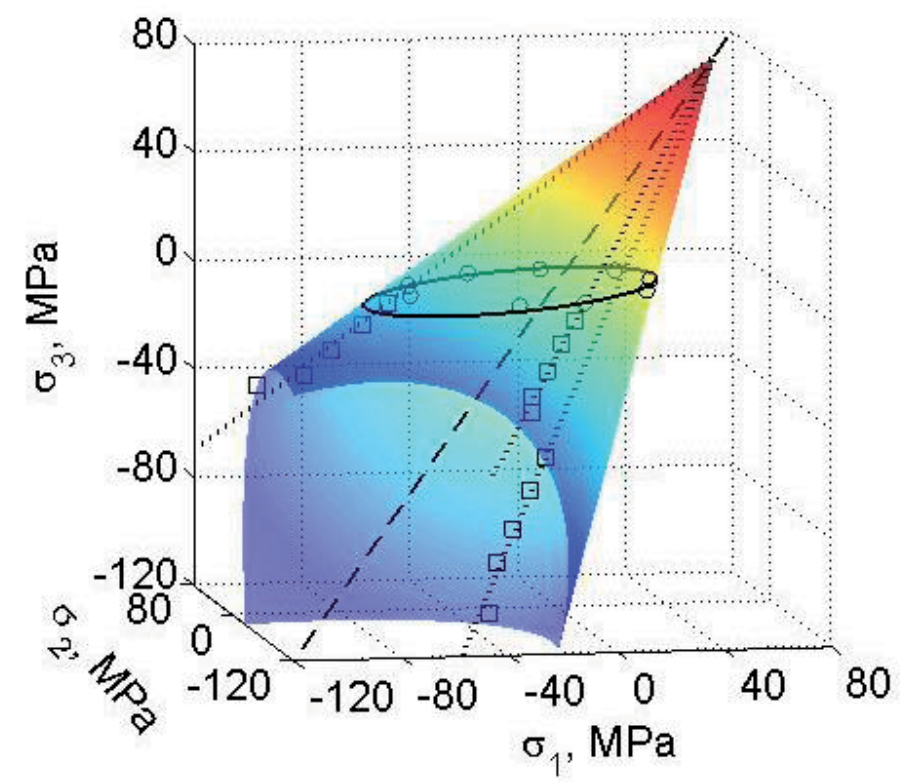

Fig. 5. Best fit Drucker-Prager yield function also indicating the hydrostatic axis and intersection with the plane-stress plane; experimental values are also depicted: ( $\square$ ) confined compression, (o) plane stress values.

\section{Conclusions}

The objective of this paper was to characterize PP in different test configurations and to apply a 3D yield criterion to predict yielding of the material in the 3D stress space. As to the determination of the yield point in various stress conditions, uniaxial and biaxial tensile tests, torsion tests, and uniaxial to confined compression tests were conducted. In order to characterize the effect of temperature, tests were performed at $-30{ }^{\circ} \mathrm{C}, 23{ }^{\circ} \mathrm{C}$ and $80{ }^{\circ} \mathrm{C}$. A linear correlation between the equivalent stress and the mean stress for all test temperatures was obtained. While the lowest equivalent stresses were obtained in the tensile regime (i.e., uniaxial and biaxial tensile tests) with positive mean tensile stresses acting upon the specimen, the highest equivalent stresses were found for the confined compression test with the largest mean compressive stresses investigated in this study. In order to calculate the pressure sensitivity index, normalized equivalent stresses were plotted against mean pressure and the pressure coefficient was calculated. As expected, the pressure dependency is seen to increase with temperature, which was mainly attributed to the free volume in the amorphous phase and the overall molecular mobility.

In order to predict the onset of yielding in the 3D stress space, the Drucker-Prager yield criterion was successfully fitted to the experimental values, with an average error between the experimentally determined yield stresses and the 3D yield criterion of about $7 \%$. The importance of adequate test methods in combination with the determination of multiaxial data for accurate material modeling of this class of materials was demonstrated. 


\section{Acknowledgements}

This research was performed at the Polymer Competence Center Leoben GmbH (PCCL, Austria) and at the Department of Aerospace Engineering and Engineering Mechanics at the University of Texas at Austin within the framework of the Kplus-program of the Austrian Ministry of Traffic, Innovation and Technology with contributions by Borealis Polyolefine GmbH. The PCCL is funded by the Austrian Government and the States Governments of Styria and Upper Austria.

\section{References}

1. M. Berer, Charakterisierung und Simulation des Verformungsverhaltens von Kunststoffrollen aus POM und PEEK (Diploma Thesis, Leoben 2007)

2. D. Stahlberg, P. Junestam, M. Johansson, E.K. Gamstedt, Prog. Org. Coat. 55 (2006)

3. M.C. Dubourg, A. Chateauminois, B. Villechaise, Tribol. Int. 36 (2003)

4. D.K. Baek, M.M. Khonsari, Wear 261 (2006)

5. P.A. Kakavas, D.P.N. Kontoni, Int. J. Numer. Methods Eng. 65 (2006)

6. J.A. Sauer, K.D. Pae, Colloid. Polym. Sci. 252 (1974)

7. J.A. Sauer, Polym. Eng. Sci. 17 (1977)

8. G. Dean, L. Crocker, Plast. Rubber Compos. 36 (2007)

9. K.D. Pae, J. Mater. Sci. 12 (1977)

10. A.Y. Gol'dman, A.B. Freidin, A.A. Lebedev, Strength Mater. 15 (1983)

11. D.C. Drucker, W. Prager, Q. Appl. Math. 10 (1952)

12. P. Bardia, R. Narasimhan, Strain 42 (2006)

13. D. Rittel, A. Dorogoy, J. Mech. Phys. Solids 56 (2008)

14. C. Feichter, Z. Major, R.W. Lang, In Proc. " $13^{\text {th }}$ International Conference on Experimental Mechanics" (2007)

15. M. Jerabek, Z. Major, R.W. Lang, Polym. Test. 29, (2010)

16. Z. Ma, K. Ravi-Chandar, Exp. Mech. 40 (2000)

17. D. Qvale, K. Ravi-Chandar, Mech. Time-Depend. Mater. 8 (2004)

18. M. Jerabek, D. Tscharnuter, Z. Major, K. Ravi-Chandar, R.W. Lang, Mech. Time-Depend. Mater. 14 (2010)

19. P.I. Vincent, Polymer 1 (1960)

20. R. Raghava, R.M. Caddell, G.S.Y. Yeh, J. Mater. Sci. 8 (1973)

21. J. Mohanray, D.C. Barton, I.M. Ward, A. Dahoun, J.M. Hiver, C. G'Sell, C. Polymer 42 (2006)

22. C. Marano, M. Rink, Mech. Time-Depend. Mater. 10 (2006)

23. C. Marano, M. Rink, Polymer 42 (2001)

24. L.M. Kachanov, Fundamentals of the Theory of Plasticity (MIR Publishers, Moscow, 1974)

25. N. W. Tschoegl, W.G. Knauss, I. Emri, Mech. Time-Depend. Mater. 6 (2002)

26. R. Quinson, J. Perez, M. Rink, A. Pavan, J. Mater. Sci. 32 (1997)

27. L.A. Fasce, V. Pettarin, C. Marano, M. Rink, P.M. Frontini, Polym. Eng. Sci. 48 (2008)

28. I.M. Ward, Mechanical Properties of Solid Polymers (John Wiley \& Sons Ltd., London, 1971) 